\title{
Apuntes sobre la institucionalización de la Medicina en Michoacán, siglo XIX
}

\author{
Notes on the institutionalization of Medicine in Michoacan, 19th century
}

Óscar A. Sánchez-Pérez y Alain R. Rodríguez-Orozco*

Facultad de Ciencias Médicas y Biológicas Dr. Ignacio Chávez, Universidad Michoacana de San Nicolás de Hidalgo, Morelia, Michoacán, México

\begin{abstract}
Resumen
El objetivo del estudio fue mostrar rasgos identitarios del proceso de institucionalización de la Medicina en el siglo XIX en Michoacán. Se señalan aspectos relevantes de la creación y la evolución de las instituciones relacionadas con el cuidado de la salud de los michoacanos durante el siglo XIX a partir de la revisión de material hemerográfico y textos referentes a la práctica de la Medicina en esa centuria, en el Estado de Michoacán.
\end{abstract}

Palabras clave: Instituciones sanitarias. Michoacán. Siglo XIX. Historia de la medicina.

\begin{abstract}
The objective of the study was to show identity traits of the process of institutionalization of Medicine in the 19th century in Michoacán. Relevant aspects of the creation and evolution of the institutions related to the health care of Michoacans during the 19th century are pointed out from the review of hemerographic material and texts referring to the practice of medicine in that century, in the State from Michoacan.
\end{abstract}

Key words: Health institutions. Michoacan. 19th century. History of medicine.

\section{Introducción}

El proceso de institucionalización de la Medicina en Michoacán durante el siglo XIX es bastante interesante, ya que a través de su estudio podemos entender la importancia histórica del discurso sanitario en la organización de la salud pública del Estado. Es importante mencionar que la historia de los organismos encargados de vigilar el ejercicio de la Medicina, su enseñanza y, en su momento, la salud pública en Morelia, fue similar a la de la máxima autoridad sanitaria de la ciudad de México. Este artículo tuvo como objetivo mostrar rasgos identitarios del proceso de institucionalización de la Medicina en el siglo XIX en Michoacán, lo cual es útil para entender los antecedentes inmediatos de las instituciones de salud que hoy operan en el Estado.

\section{Creación de instituciones para atender la salud de los michoacanos en el siglo XIX}

A inicios del decimonónico, los ayuntamientos debían contar con una comisión encargada especialmente de

\section{Correspondencia:}

*Alain R. Rodríguez-Orozco

Rafael Carrillo s/n

Col. Centro

Fecha de recepción: 02-09-2020

C.P. 58020 , Morelia, Mich., México

E-mail: alain.rodriguez@umich.mx

DOI: 10.24875/CIRU.20000970
Cir Cir. 2021;89(3):420-425

Contents available at PubMed www.cirugiaycirujanos.com 0009-7411/@ 2020 Academia Mexicana de Cirugía. Publicado por Permanyer. Este es un artículo open access bajo la licencia CC BY-NC-ND (http://creativecommons.org/licenses/by-nc-nd/4.0/). 
procurar la sanidad del lugar, la cual debía contar siempre con el apoyo de un párroco y uno o más facultativos donde los hubiere, a fin de ilustrarla en sus deliberaciones. Sin embargo, el estado sanitario continuó en deterioro, ya que aunque las comisiones existían en la mayoría de los municipios del Estado de Michoacán tendían a actuar solo en tiempos de epidemia'.

El Protomedicato en Michoacán fue creado en 1829 por los médicos Juan Manuel González Urueña y Juan Francisco Macouzet, y el cirujano Francisco Córdova, como un intento por tener en Michoacán una institución que tuviera jurisdicción en todo el Estado. Sus funciones principales estuvieron orientadas al control del ejercicio médico y a la promoción de acciones de salud en caso de epidemias. Su atribución más importante era la tarea de examinar a quienes intentaban ejercer actividades relacionas con la salud, como la medicina, la cirugía, la farmacia y la flebotomía'.

Los integrantes del Protomedicato examinaron el problema social de la salud y concluyeron que una de las principales causas de la deficiente atención era la falta de personas preparadas para ejercer la Medicina en el Estado. Como solución propusieron el establecimiento de una Cátedra de Medicina y la elaboración de un reglamento para la erección y el funcionamiento de la misma ${ }^{2}$. El 9 de noviembre de 1829 se decretó el establecimiento de la cátedra, a la cual se facilitarían los recursos económicos suficientes para el pago de un profesor, el instrumental necesario y los gastos que surgieran para su mantenimiento. Se determinó también que el Protomedicato estaría a cargo de reglamentar y asegurar el buen funcionamiento de la Cátedra de Medicina ${ }^{3}$.

Para el 21 de noviembre del mismo año se establecieron los siguientes puntos: «1) La Cátedra de Medicina estará bajo la protección inmediata del gobierno del Estado; 2) La Cátedra comprenderá en su estudio el de la Anatomía Descriptiva, la Fisiología, Patología General e Higiene Pública y Privada; 3) Los cursos, que serán tres, comenzarán el 15 de enero y terminarán el último de noviembre; 4) Serán lectivos todos los días, exceptuando solamente los domingos y festividades religiosas o políticas; 5) Habrá dos horas de cátedra por la mañana y de siete a nueve y una en la tarde cuando el catedrático lo tuviere por conveniente; 6) El primero y segundo curso se destinarán para la enseñanza de la Anatomía y Fisiología, en el tercero se enseñará la Patología e Higiene; 7) los Autores por quienes se debe estudiar serán aprobados por el Protomedicato» ${ }^{3}$.
La Cátedra de Medicina no fue inaugurada en la fecha que se había establecido, sino el 1 de mayo de 1830. El 3 de marzo de 1834, el Protomedicato fue transformado en la Facultad Médica de Michoacán. En el decreto por el cual se establece la Facultad se estableció también la unión de las "ciencias médica y quirúrgica», y los aprendizajes de ambas disciplinas fueron dirigidos por la Facultad Médica. A diferencia del organismo anterior, en ese momento quedaría conformada por dos profesores médico y cirujano, un farmacéutico, un fiscal y un secretario. Las tareas que estaban a cargo de la Facultad Médica eran examinar a aquellos que pretendían ejercer la medicina y vigilar que ejercieran correctamente la profesión, encargarse de que fueran sancionados aquellos que practicaran la medicina sin un título profesional, elaborar farmacopeas, la vigilancia de las boticas y de la calidad de los alimentos y bebidas, y atender que se realizara la limpieza de las calles, plazas, panteones, hospitales y hospicios ${ }^{1}$.

Otra de las tareas encomendadas a la Facultad Médica de Michoacán fue la creación de un código de leyes sanitarias y un reglamento para el Estado'. Las intervenciones por parte de los estadounidenses y franceses obstaculizaron aún más la posibilidad de que la Facultad Médica y cualquier institución de instrucción pudiera realizar las tareas para las que habían sido creadas ${ }^{4}$.

Durante el segundo imperio hubo esfuerzos por mejorar la salud pública. Se organizó un Consejo Central de Salubridad que fue publicado en la Ley sobre la Policía General del Imperio en 1865, y también se tomaron en cuenta otros aspectos, como las Juntas Subalternas y las de Sanidad'. Después del triunfo del gobierno republicano, la Facultad Médica fue suprimida para establecer en su lugar la Junta de Salubridad de Michoacán el 3 de enero de 1869, la cual era renovable cada año. Estaba compuesta por cinco vocales y tenía las tareas de vigilar el ejercicio de la medicina, «practicar los exámenes, visitar las boticas, cuidar de la higiene y la salubridad pública, formar una Farmacopea y coleccionar, bajo forma de Código, las leyes de policía médica y sanitaria, y ayudar a la justicia con sus investigaciones ${ }^{5}$. La Junta estaba conformada por dos tipos de comisiones: las permanentes, que tenían funciones establecidas de acuerdo para lo que habían sido creadas, como las comisiones de medicina legal, estadística patológica e higiene pública, y las comisiones «accidentales", que eran creadas de acuerdo con las circunstancias'. 
En el año 1894, la Junta de Salubridad fue sustituida por el Consejo Superior de Salubridad, establecido por ley el 12 de junio de 1894. Esta reorganización de la salud en Morelia fue explicada por el gobierno de la siguiente manera ${ }^{6}$ :

«Mas para conservar la salubridad pública, es necesario atender con empeño y eficacia á cada uno de los elementos que la constituyen, y como estos son múltiples y en su mayor parte de difícil arreglo, y por los conocimientos facultativos que suponen, ya por los intereses y derechos que entran en juego en la combinación de tales elementos es indispensable hacer de la salubridad un ramo administrativo especial, encargado á un cuerpo consultor que tenga los elementos de ciencia y autoridad que sean necesarios para la disposición armónica de todos los pormenores.»

El nuevo órgano encargado de la salubridad pública no parecía tener cambios muy notorios respecto a su precedente y no representó un cambio radical para el mejoramiento de la salubridad, ya que el cambio sustancial que había entre la Junta de Salubridad y el Consejo fue que los miembros de aquella Junta desempeñaban su cargo de manera gratuita mientras que los del Consejo percibirían la retribución que les asignaría el Estado según el presupuesto de egresos. El cambio parecía no ser muy grande, pero la intervención del Estado tenía como principal objetivo el mejoramiento del servicio público, pues por grande que fuera el empeño de los miembros de la Junta en cumplir con los deberes de sus cargos no habrían puesto nunca la atención suficiente debido a que estos cargos no tenían una remuneración económica, por lo que la mayor atención de sus miembros estaba en aquellas tareas que les permitían obtener ingresos seguros ${ }^{6}$. Y así, con uno de los elementos más motivantes para realizar una tarea asignada, un buen sueldo, es que se pretendía mejorar el rendimiento de los miembros del Consejo Superior de Salubridad, en quienes recaería todo lo relativo a la higiene y la salubridad pública, así como el estudio de las causas de las enfermedades dominantes, epidémicas y epizootias para proponer las medidas convenientes; la vigilancia de la calidad de las bebidas y comestibles, el aseo de las poblaciones y expedir los reglamentos que fuesen necesarios para mejorar la salubridad pública ${ }^{6}$.

Una de las tareas más importantes que se comisionarían al Consejo Superior de Salubridad sería la realización de un código sanitario para el Estado de
Michoacán de Ocampo. El proyecto del código, así como el reglamento bajo el que se regiría el Consejo, fueron emitidos un año después de su instauración. En la primera parte del reglamento, publicado el 5 de enero de 1896, se menciona lo siguiente ${ }^{7}$ :

Art. $1 .^{\circ}$. Desde el 1 de Enero de 1896, el Consejo Superior de Salubridad se compondrá de cinco miembros propietarios y cuatro suplentes. Tanto entre los propietarios como entre los suplentes habrá un Profesor de Farmacia, siendo los restantes Profesores de Medicina. Habrá además un inspector de sanidad, cuyas funciones determinarán los reglamentos respectivos.

Art. $2{ }^{\circ}$. El Presidente y vocales del Consejo serán nombrados por el Gobierno y durarán ejerciendo su encargo dos años contados desde el $1 .^{\circ}$ de Enero del año en que empiecen á funcionar.

Art. 3.․ El Presidente del Consejo asistirá á la oficina todos los días á la hora que se designe el Reglamento, para hacer el despacho de los asuntos pendientes de acuerdo.

Art. $4 .{ }^{\circ}$. Los miembros del Consejo, con excepción del Profesor de Farmacia, tendrán á su cargo la asistencia médica de los establecimientos públicos que á cada uno asigne el Ejecutivo.

Art. 5.․ Las visitas ordinarias se harán cuando menos dos veces cada semana en los días y horas que designe el Gobierno, sin perjuicio de que los médicos ocurran en cualquier otro día é inmediatamente á la hora que se les llame para presentar sus auxilios á los enfermos que los necesiten.

Art. $6{ }^{\circ}$. Cuando amenace ó se inicie alguna epidemia en los establecimientos de que se trata, ó en cualquiera otro caso en que por razones de higiene los médicos lo estimen necesario, darán inmediato aviso al Consejo para que este Cuerpo determine ó promueva lo que fuere conveniente á fin de evitar ó combatir el mal.

Art. $7 .^{\circ}$. El día último de cada mes los directores ó encargados de los establecimientos y el Prefecto del Distrito, por lo que ve á las fuerzas de seguridad pública, informarán por escrito al Gobierno del resultado de las visitas practicadas y del estado de sanidad en que se encuentren las personas que están bajo su vigilancia.

Art. 8.․ Igual informe darán los médicos al Consejo, para que en vista de aquél dicte dicho Cuerpo las determinaciones que crea necesarias para 
el mejoramiento de la salubridad en los lugares visitados.

Art. $9^{\circ}$. Los sueldos de que disfruten los miembros y empleados del Consejo serán los que determine el presupuesto, percibiendo en el segundo semestre del presente año fiscal los que en acuerdo de esta misma fecha ha señalado el Ejecutivo.

En una segunda parte se mencionan cuáles son las atribuciones específicas con las que debe cumplir el Consejo Superior de Salubridad:

I. Iniciar ante el Gobierno, á la brevedad posible, un Código Sanitario y los reglamentos que de él deban emanar.

II. Vigilar por el extricto complimiento de las prescripciones del Código y reglamentos que refiere el anterior inciso, dando cuenta al Gobierno de las infracciones que se noten, para que ponga el remedio que corresponda.

III. Prácticar en los términos que disponga el código Sanitario las visitas que fueren necesarias á las habitaciones, establecimientos y edificios peligrosos, incómodos é insalubres; á las bóticas, expendios de bebidas y comestibles; á los mercados, teatros, talleres, fabricas, fondas, escuelas, colegios, cuarteles, cárceles, hospicios, hospitales, casas de tolerancia, abastos, establos, zahurdas, panteones, y en general á todo cuanto tenga aglomeración peligrosa de personas ó animales, ó pueda constituir un amaro á la salubridad.

IV. Recabar de quienes estime conveniente las noticias que sean necesarias para conocer la salubridad del Estado.

V. Organizar el servicio sanitario en los términos que disponga el código referido.

VI. Resolver las consultas que sobre las materias de su resorte le hicieren las autoridades del Estado. Dichas consultas se harán por conducto del Gobierno, si proceden de autoridades del orden administrativo, y por conducto del Tribunal Superior de Justicia si provienen del poder judicial.

VII.Indicar oportunamente al Ejecutivo para los efectos consiguientes, las substancias alimenticias, bebidas ó drogas cuya introducción al Estado pueda ser nociva á la salubridad.

VIII.Indicar asimismo cuando una epidemia ó epizootia amenace extenderse á Michoacán, á fin de que para evitarlo dicte las medidas conducentes.
IX. Formar y publicar al fin de cada año la Estadística Médica de la Capital, con una noticia de las enfermedades reinantes, endémicas y epizootias que hubieren aparecido, haciendo constar cuáles sean las causas y los datos que puedan ilustrarla; así como un resumen de las noticias recibidas de las demás poblaciones del Estado.

X. Informar al Gobierno de los motivos de insalubridad que se encuentren en las habitaciones, establecimientos y demás que refiere la $3^{3}$ fracción del presente artículo, así como de los que se adviertan en los basureros, acequias, canales, atarjeas y caños, á efecto de que se dicten las medidas necesarias que deberá indicar el mismo Consejo.

XI. Cuidar del exacto cumplimiento de las leyes y disposiciones relativas á la vacunación, dando cuenta al Gobierno de las infracciones que se noten, para que ponga el remedio que corresponda.

XII.Conservar hasta donde sea posible las otras vacunas preventivas conocidas y las que se encuentren conocidas y las que se encuentren eficaces para impedir el desarrollo de las enfermedades.

XIII. Ponerse, hasta donde fuere posible, en relación con las Juntas de Salubridad del país y con las otras Corporaciones análogas del extranjero.

Se propuso la división en comisiones que tendrían diversas tareas para facilitar la observación de la higiene pública, tales como la comisión de admiración y reglamentación de personal sanitario, la comisión de sanidad en los Distritos, de alimentos y bebidas, de Farmacia, droguerías, boticas y otros establecimientos, de epidemiología y de epizootias entre otras, que serían un total de veintidós comisiones $^{9}$. El Consejo Superior de salubridad se encaminaría hacia la «modernización» sanitaria, y como prueba de ello se expediría el primer Código Sanitario de Michoacán, que entraría en vigor el 1 de enero de 1896.

Hubo varias figuras destacadas ligadas a la Medicina en Michoacán en el siglo XIX, pero ninguna con tanto realce como el Dr. José Manuel González Urueña, ilustrísimo hombre de ciencias y humanidades, nacido en Tancítaro, Michoacán, en 1798, y que entre sus múltiples logros fue fundador de la Escuela Médica de Michoacán, destacadísimo defensor del Colegio de San Nicolás, Primer Presidente del Protomedicato de 
Michoacán, Presidente de la Facultad Médica, magnífico escritor y traductor, y médico de enorme calidad científica y humana en su época; y en la política fue diputado local y federal, senador, consejero de gobierno y gobernador. El padre de la Escuela Médica de Michoacán murió en Celaya, Guanajuato, en 1854, tras un inmerecido destierro forzado por el régimen de López de Santa Anna ${ }^{10}$.

Además del Hospital San Juan de Dios en Morelia, que se transformaría posteriormente en Hospital Civil, en el Estado de Michoacán se inauguraron o continuaron en operación los siguientes hospitales en el siglo XIX10:

- Hospital Civil de Pátzcuaro, que en 1860 pasó de los juaninos al gobierno del Estado, una evolución similar a la de su homónimo en Morelia.

- Hospital de Zamora, que se considera continuación de uno fundado por Vasco de Quiroga. En 1863, el general José López Uraga usó parte de sus recursos en la guerra contra los franceses y luego pasa al control del Estado.

- Hospital eclesiástico de Zamora (1841).

- Hospital de Tacámbaro (1857), que se transforma en Hospital Civil en 1877.

- Hospital de La Piedad (1882).

- Hospital Civil de Uruapan (1890).

- Hospital de Cotija (1895).

- Hospital de Zitácuaro (1896).

- Hospital de Puruándiro (1897) ${ }^{10}$.

Merece la pena nombrar las agrupaciones médicas que se establecieron en Morelia, Michoacán, durante el siglo XIX y que tuvieron en común vidas cortas y ausencia de material reconocido que documente su accionar o sus memorias. Ellas son, según Romero-Flores (1935), la Sociedad Médica de Michoacán, fundada en 1869 y dirigida por el Dr. Gabino R. Mota, la cual operó en el Colegio de San Nicolás; la Academia de Estudios Médicos, integrada por estudiantes de Medicina (1874-1875), que se reunió en el antiguo colegio jesuita de Michoacán; y dos instituciones con el nombre de Academia de Medicina que operaron en 1876, una constituida por un grupo de personas que se reunía en casa de Don Ignacio Torres (1876) y otra presidida por el Dr. Mateo González y que sesionó en el Colegio de San Nicolás, a la que asistían alumnos y profesores de la Escuela de Medicina $^{10}$.

La tabla 1 detalla aspectos relevantes de la creación y la evolución de las instituciones relacionadas con la Medicina en Michoacán durante el siglo $\mathrm{XIX} \mathrm{X}^{4,10-12}$.
Tabla 1. Cronología. Aspectos relevantes de la creación y la evolución de las instituciones relacionadas con la Medicina en Michoacán, en el siglo XIX

1826 Creación de la Junta Médico Quirúrgica de Michoacán

1829 Sustituye a la Junta el Protomedicato de Michoacán

1830 Fundación de la Cátedra de Medicina en el Hospital de San Juan de Dios con miembros del Protomedicato como docentes y como presidente Juan Manuel González Ureña

1833 Reforma del plan de estudios. Se unen las carreras de Medicina y Cirugía en una misma institución, el Instituto/ Establecimiento Médico Quirúrgico

1834 Se transforma el protomedicato en Facultad Médica de Michoacán

1847 Se reabre el Colegio de San Nicolás y queda como sede de los estudios de Medicina en el Estado

1848 Se habilitan cursos para parteras bajo la conducción del Dr. Joaquín Mota, quien también gratuitamente fue responsable de la Cátedra de Química, al igual que don Manuel Urbizo lo hizo con la Cátedra de Farmacia y Botánica. Los estudiantes de medicina comienzan a recibir clases en el Colegio de San Nicolás

1849 Se abre la Cátedra de Patología Externa para los estudios de Medicina en el Colegio de San Nicolás, dirigida gratuitamente por don Vicente Franco

1850 Se clausuran los estudios de Medicina por problemas en la calidad de la enseñanza, como falta de laboratorios y de personal

1858 Secularización. El Hospital de San Juan en Morelia, coordinado por religiosos, pasa a ser Hospital Civil bajo el mando del gobierno del Estado

1859 Reapertura de los estudios de Medicina con sede en el Colegio de San Nicolás con el plan de estudios de 1843; se usan para la enseñanza el Hospital Civil y un Coliseo

1861 El Hospital Civil de Morelia se traslada al Antiguo Convento de San Diego

1863 El Hospital Civil de Morelia se traslada al antiguo Convento de la Merced. Cierre de las actividades académicas de Medicina y del Colegio de San Nicolás por la invasión francesa

1865 Se constituye el Consejo Central de Salubridad

1867 El Hospital Civil de Morelia se traslada al exconvento de monjas Capuchinas. Restablecimiento de actividades en el Colegio de San Nicolás por indicaciones del gobierno republicano

1869 La Facultad Médica es reemplazada por la Junta de Salubridad del Estado de Michoacán

1881 Reforma profunda del plan de estudios de la carrera de Medicina en el Colegio de San Nicolás

1886 Aprobación del primer reglamento para clínicas y la enseñanza práctica se dicta en el Hospital Civil de Morelia. Se establece el Museo Michoacano con Nicolás León como director 
Tabla 1. Cronología. Aspectos relevantes de la creación y la evolución de las instituciones relacionadas con la Medicina en Michoacán, en el siglo XIX (Continuación)

1894 La Junta de Salubridad es sustituida por el Consejo Superior de Salubridad. Se formaban en Morelia médicos, farmacéuticos, parteras, flebotomistas, dentistas y profesores de pequeña cirugía

1895 Queda constituida la Escuela Médica del Estado de Michoacán, anexa al Colegio de San Nicolás y al Hospital Civil, y comienza sus actividades en 1896 de acuerdo con la Ley Orgánica de la Instrucción Secundaria y Profesional (1894)

\section{Conclusiones}

Se realizó una revisión de las instituciones relacionadas con la práctica de la Medicina en Michoacán en el siglo XIX, atendiendo al surgimiento, las funciones y la evolución de las más importantes, que fueron, en orden cronológico, el Protomedicato, la Facultad Médica y la Cátedra de Medicina, el Colegio de San Nicolás, el Hospital San Juan de Morelia (que luego se transforma en Hospital Civil), el Consejo Central de Salubridad y la Junta de Salubridad. Además, se señalan los hospitales que hubo en Michoacán durante el siglo XIX; muchos de ellos fueron inicialmente dirigidos por religiosos y pasaron a ser controlados por el Estado, transformados en hospitales civiles. Por último, se señalan las agrupaciones médicas estatales integradas por médicos o estudiantes de Medicina, de las que son destacables sus cortas existencias y la ausencia, hasta el momento, de registros que documentaran su accionar. A fines del siglo XIX, el Estado contaba con una red de instituciones por él manejadas, se había establecido la regulación de la práctica médica y se contaba con una escuela cuyo objetivo era formar profesionales en Medicina y Cirugía, y esta era orientada por médicos capacitados, en los recintos del Colegio de San Nicolás, en el que se realizó una profunda revisión del plan de estudios en 1881 para adaptarlo a las nuevas exigencias de la práctica clínica, y las prácticas se realizaban en el Hospital Civil de Morelia, que fue el primer hospital universitario del Estado con un reglamento para clínicas y enseñanza práctica de la Medicina, establecido en 1886.

\section{Responsabilidades éticas}

Protección de personas y animales. Los autores declaran que para esta investigación no se han realizado experimentos en seres humanos ni en animales.

Confidencialidad de los datos. Los autores declaran que en este artículo no aparecen datos de pacientes.

Derecho a la privacidad y consentimiento informado. Los autores declaran que en este artículo no aparecen datos de pacientes.

\section{Financiamiento}

Los autores no recibieron patrocinio para llevar a cabo este artículo.

\section{Conflicto de intereses}

Los autores declaran no tener conflicto de intereses alguno.

\section{Bibliografía}

1. Zavala Ramírez MC. El arte de conservar la salud en el Porfiriato. Universidad Michoacana de San Nicolás de Hidalgo, Morelia, Michoacán; 2010. p. 38-9.

2. Sánchez Díaz G. Las contribuciones michoacanas a la Medicina y la Salud Pública en el siglo XIX. En: Martínez Cortés F, Guzmán Ávila JN, coordinadores. Ensayos sobre Historia de la Medicina. Instituto de Investigaciones Históricas, Universidad Michoacana de San Nicolás de Hidalgo; 2003. p. 129-30.

3. Martínez Cortés F, Guzmán Ávila N, coordinadores. Ensayos sobre Historia de la Medicina. Instituto de Investigaciones Históricas, Universidad Michoacana de San Nicolás de Hidalgo, Morelia; 2003.

4. León N. Historia de la Medicina en Michoacán. Gobierno del Estado de Michoacán de Ocampo; 1984.

5. Flores y Troncoso F. Historia de la Medicina en México desde la época de los indios hasta el presente. México: Instituto Mexicano del Seguro Social; 1982. p. 219.

6. Periódico Oficial del Gobierno del Estado de Michoacán de Ocampo, Tomo II, Núm. 50, México-Morelia, domingo 24 de junio de 1894, p. 3.

7. Periódico Oficial del Gobierno del Estado de Michoacán de Ocampo, Tomo IV, Núm. 2, México-Morelia, domingo 5 de enero de 1896, p. 3.

8. Periódico Oficial del Gobierno del Estado de Michoacán de Ocampo, Tomo IV, Núm. 9, jueves 30 de enero de 1896, p. 5.

9. Periódico Oficial del Gobierno del Estado de Michoacán de Ocampo, Tomo IV, Núm. 10, domingo 2 de febrero de 1896, p. 5.

10. Romero-Flores J. Historia de la Escuela de Medicina en Michoacán. En: Anales del Museo Nacional de México 1935, No. 2, p. 351-459.

11. Ballesteros-Olivares H. 175 años de la Facultad de Ciencias Médicas y Biológicas "Dr Ignacio Chávez" de la Universidad Michoacana. Bol Mex His Fil Med. 2006;9:21-30.

12. Figueroa Zamudio S. La cátedra de Medicina en Michoacán en el siglo XIX. En: Alvarado ML, Pérez Puente L, coordinadores. Cátedras y catedráticos en la historia de las universidades e instituciones de educación superior en México. I. La educación colonial. México: IISUE-UNAM; 2008. p. 339-363. Disponible en: http://132.248.192.241:8080/jspui/bitstream/IISUE_UNAM/38/1/La\%20c\%C3\%A1tedra\%20de\%20medicina\%20en\%20Michoac\%C3\%A1n\%20en\%20el\%20siglo\%20XIX.pdf 